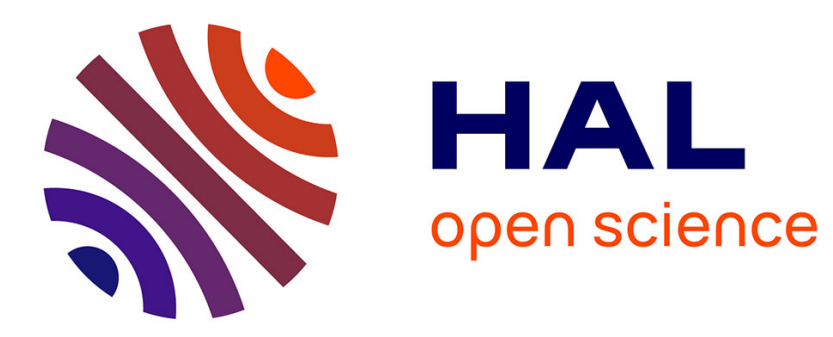

\title{
Elementary Examination of NeutroAlgebras and AntiAlgebras viz-a-viz the Classical Number Systems
}

\author{
A a A Agboola, Muritala A Ibrahim, E O Adeleke
}

\section{To cite this version:}

A a A Agboola, Muritala A Ibrahim, E O Adeleke. Elementary Examination of NeutroAlgebras and AntiAlgebras viz-a-viz the Classical Number Systems. International Journal of Neutrosophic Science, 2020, 4, pp.16 - 19. 10.5281/zenodo.3752896 . hal-02924593

\section{HAL Id: hal-02924593 \\ https://hal.science/hal-02924593}

Submitted on 1 Sep 2020

HAL is a multi-disciplinary open access archive for the deposit and dissemination of scientific research documents, whether they are published or not. The documents may come from teaching and research institutions in France or abroad, or from public or private research centers.
L'archive ouverte pluridisciplinaire HAL, est destinée au dépôt et à la diffusion de documents scientifiques de niveau recherche, publiés ou non, émanant des établissements d'enseignement et de recherche français ou étrangers, des laboratoires publics ou privés. 


\title{
Elementary Examination of NeutroAlgebras and AntiAlgebras viz-a-viz the Classical Number Systems
}

\author{
${ }^{1}$ A.A.A. Agboola[ ${ }^{2}$ M.A. Ibrahim and ${ }^{3}$ E.O. Adeleke \\ ${ }^{1,2,3}$ Department of Mathematics, Federal University of Agriculture, Abeokuta, Nigeria. \\ agboolaaaa@funaab.edu.ng ${ }^{1}$,muritalaibrahim40@gmail.com ${ }^{2}$,yemi376@yahoo.com ${ }^{3}$.
}

\begin{abstract}
The objective of this paper is to examine NeutroAlgebras and AntiAlgebras viz-a-viz the classical number systems.
\end{abstract}

Keywords: NeutroAlgebra, AntiAlgebra, NeutroAlgebraic Structure, AntiAlgebraic Structure.

\section{Introduction}

The notions of NeutroAlgebra and AntiAlgebra were recently introduced by Florentin Smarandache ${ }^{1}$ Smarandache in ${ }^{2}$ revisited the notions of NeutroAlgebra and AntiAlgebra and in ${ }^{3}$ he studied Partial Algebra, Universal Algebra, Effect Algebra and Boole's Partial Algebra and showed that NeutroAlgebra is a generalization of Partial Algebra. In the present Short Communication, we are going to examine NeutroAlgebras and AntiAlgebras viz-a-viz the classical number systems. For more details about NeutroAlgebras, AntiAlgebras, NeutroAlgebraic Structures and AntiAlgebraic Structures, the readers should see $!^{1}+3$

Let $U$ be a universe of discourse and let $X$ be a nonempty subset of $U$. Suppose that $A$ is an item (concept, attribute, idea, proposition, theory, algebra, structure etc.) defined on the set $X$. By neutrosophication approach, $X$ can be split into three regions namely: $\langle A\rangle$ the region formed by the sets of all elements where $\langle A\rangle$ is true with the degree of truth (T), $<$ anti $A>$ the region formed by the sets of all elements where $\langle A>$ is false with the degree of falsity $(\mathrm{F})$ and $<$ neut $A>$ the region formed by the sets of all elements where $\langle A\rangle$ is indeterminate (neither true nor false) with the degree of indeterminacy (I). It should be noted that depending on the application, $\langle A\rangle$, $\langle$ anti $A\rangle$ and $\langle$ neut $A\rangle$ may or may not be disjoint but they are exhaustive that is; their union is $X$. If $A$ represents Function, Operation, Axiom, Algebra etc, then we can have the corresponding triplets $<$ Function, NeutroFunction, AntiFunction $>$, $<$ Operation, NeutroOperation, AntiOperation $>,<$ Axiom, NeutroAxiom, AntiAxiom $>$ and $<$ Algebra, NeutroAlgebra, AntiAlgebra $>$ etc.

Definition 1.1. 1

(i) A NeutroAlgebra $X$ is an algebra which has at least one NeutroOperation or one NeutroAxiom that is; axiom that is true for some elements, indeterminate for other elements, and, false for other elements.

(ii) An AntiAlgebra $X$ is an algebra endowed with a law of composition such that the law is false for all the elements of $X$.

Definition 1.2. ${ }^{1}$ Let $X$ and $Y$ be nonempty subsets of a universe of discourse $U$ and let $f: X \rightarrow Y$ be a function. Let $x \in X$ be an element. We define the following with respect to $f(x)$ the image of $x$ :

(i) Inner-defined or Well-defined: This corresponds to $f(x) \in Y$ (True)(T). In this case, $f$ is called a Total Inner-Function which corresponds to the Classical Function.

(ii) Outer-defined: This corresponds to $f(x) \in U-Y$ (Falsehood) (F). In this case, $f$ is called a Total Outer-Function or AntiFunction.

(iii) Indeterminacy: This corresponds to $f(x)=$ indeterminacy (Indeterminate) (I); that is, the value $f(x)$ does exist, but we do not know it exactly. In this case, $f$ is called a Total Indeterminate Function.

Corresponding Author: A.A.A. Agboola aaaola2003@yahoo.com 


\section{Subject Matter}

In what follows, we will consider the classical number systems $\mathbb{N}, \mathbb{Z}, \mathbb{Q}, \mathbb{R}, \mathbb{C}$ of natural, integer, rational, real and complex numbers respectively and noting that $\mathbb{N} \subseteq \mathbb{Z} \subseteq \mathbb{Q} \subseteq \mathbb{R} \subseteq \mathbb{C}$. Let,,$+- \times, \div$ be the usual binary operations of addition, subtraction, multiplication and division of numbers respectively. Using elementary approach, we will examine whether or not the abstract systems $(\mathbb{N}, *),(\mathbb{Z}, *),(\mathbb{Q}, *),(\mathbb{R}, *),(\mathbb{C}, *)$ are NeutroAlgebras or and AntiAlgebras where $*=+,-, \times, \div$.

(1) Let $X=\mathbb{N}$.

(i) It is clear that $(X,+)$ and $(X, \times)$ are neither NeutroAlgebras nor AntiAlgebras.

(ii) For some $x, y \in X, x-y \in X$ (True) (Inner) or $x-y \notin X$ (False) (Outer). However, for all $x, y \in X$ with $x \leq y, x-y \notin X$ (False) (Outer) and for all $x, y \in X$ with $x>y$, we have $x-y \in X$ (True) (Inner). This shows that - is a NeutroOperation over $X$ and $\therefore(X,-)$ is a NeutroGroupoid. The operation - is not commutative for all $x \in X$. This shows that - is AntiCommutative over $X$. We claim that - is NeuroAssociative over $X$.

Proof. For $x>y, z=0$, we have $x-(y-z)=(x-y)-z$, or $x-y+0=x-y-0>0$ (degree of Truth) (T). However, for $x>y, z \neq 0$, we have $x-(y-z) \neq(x-y)-z$ (degree of Falsehood) (F). For $x<y, c=0$, we have $x-y+0=x-y-0<0$ (degree of Indeterminacy) (I). This shows that - is NeutroAssociative and $\therefore(X,-)$ is a NeutroSemigroup.

(iii) For all $x \in X, x \div 1 \in X$ (True) (Inner). For some $x, y \in X, x \div y \notin X$ (False) (Outer). However, if $x$ is a multiple of $y$ including 1 , then $x \div y \in X$ (True) (Inner). This shows that $\div$ is a NeutroOperation and therefore, $(X, \div)$ is a NeutroGroupoid. It can be shown that $\div$ is NeutroAssociative over $X$ and therefore, $(X, \div)$ is a NeutroSemigroup.

The equation $a x=b$ is not solvable for some $a, b \in X$. However, if $b$ is a multiple of $a$ including 1 , then the equation is solvable and the solution is called a NeutroSolution. Also, the equation $a c x^{2}+b d=$ $(a d+b c) x$ is not solvable for some $a, b, c, d \in X$. However, if $b$ is a multiple of $a$ including 1 and $c$ is a multiple of $d$ including 1 , the equation is solvable and the solutions are called NeutroSolutions.

Let $\circ$ be a binary operation defined for all $x, y \in X$ by

$$
x \circ y=\left\{\begin{array}{rll}
0 & \text { if } x=y \\
-\alpha & \text { if } x<y \\
-\beta & \text { if } x>y
\end{array}\right.
$$

where $\alpha, \beta \in \mathbb{N}$ such that $\alpha \leq \beta$. It is clear that $\circ$ is an AntiOperation on $X$ and $\therefore(X, \circ)$ is an AntiAlgebra.

(2) Let $X=\mathbb{Z}$.

(i) $(X,+)$ and $(X, \times)$ are neither NeutroAlgebras nor AntiAlgebras.

(ii) For all $x, y, z \in X$ such that $x, y=0,1$, we have $x-y=y-x=0 \in X$ (True), otherwise for other elements, the result is False (Outer) so that - is NeutroCommutative over $X$. However,if $x, y, z=0$, then $x-(y-z)=(x-y)-z=0 \in X$ (True), otherwise for other elements, the result is False and consequently, - is NeutroAssociative over $X$ and hence $(X,-)$ is a NeutroSemigroup.

(iii) For all $x \in X, x \div \pm 1 \in X$ (True) (Inner). For all $x \in X, x \div 0=$ indeterminate (Indeterminacy). For some $x, y \in X, x \div y \notin X$ (False) (Outer) however, if $x$ is a multiple of $y$ including \pm 1 , then $x \div y \in X$ (True) (Inner). This shows that $\div$ is a NeutroOperation over $X$ and $\therefore(X, \div)$ is a NeutroGroupoid. It can also be shown that $(X, \div)$ is a NeutroSemigroup.

The equation $a x=b$ is not solvable for some $a, b \in X$. If $a=0$, the solution is indeterminate (Indeterminacy). However, if $b$ is a multiple of $a$ including \pm 1 , then the equation is solvable and the solution is called a NeutroSolution. Also, the equation $a c x^{2}+(a d-b c) x-b d=0$ is not solvable for some $a, b, c, d \in X$. However, if $b$ is a multiple of $a$ including \pm 1 and $c$ is a multiple of $d$ including \pm 1 , the equation is solvable and the solutions are called NeutroSolutions.

For all $x, y \in X$, let $\circ$ be a binary operation defined by $x \circ y=\ln (x y)$. If $x, y=0$, we have $x \circ y=$ indeterminate (Indeterminacy) (I). If $x>0, y<0$, we have $x \circ y=$ indeterminate (Indeterminacy) (I). If $x>0, y>0$, we have $x \circ y=$ False (F) except when $x=y=1$. These show that $\circ$ is a NeutroOperation over $X$ and $\therefore(X \circ)$ is a NeutroAlgebra. 
Let $\circ$ be a binary operation defined for all $x, y \in X$ by

$$
x \circ y=\left\{\begin{aligned}
-1 / 2 & \text { if } x<y \\
1 / 2 & \text { if } x>y
\end{aligned}\right.
$$

It is clear that $\circ$ is an AntiOperation on $X$ and $\therefore(X, \circ)$ is an AntiAlgebra.

(3) Let $X=\mathbb{Q}$.

(i) $(X,+)$ and $(X, \times)$ are neither NeutroAlgbras nor AntiAlgebras.

(ii) For all $x, y, z \in X$ such that $x, y, z=1$, we have $x-y=y-x=0 \in X$ (True), otherwise for other elements, the result is False so that - is NeuroCommutative over $X$. Also,if $x, y, z=0$, then $x-(y-z)=(x-y)-z=0 \in X$ (True), otherwise for other elements, the result is False and consequently, - is NeutroAssociative over $X$ and $(X,-)$ is a NeutroSemigroup.

(iii) For all $0 \neq x, y \in X, x \div y \in X$ (True) (Inner) but for all $x \in X, x \div 0=$ indeterminate (Indeterminacy). $\therefore(X, \div)$ is a NeutroAlgebra which we call a NeutroField.

For all $x, y \in X$, let $\circ$ be a binary operation defined by $x \circ y=e^{x \div y}$. If $x, y=0$, we have $x \circ y=$ indeterminate (Indeterminacy) (I). If $x>0, y=0$, we have $x \circ y=$ indeterminate (Indeterminacy) (I). If $x>0, y>0$, we have $x \circ y=$ False $(\mathrm{F})$. These show that $\circ$ is a NeutroOperation over $X$ and $\therefore(X \circ)$ is a NeutroAlgebra.

Let $\circ$ be a binary operation defined for all $x, y \in X$ by

$$
x \circ y=\left\{\begin{aligned}
-e & \text { if } x \leq y \\
e & \text { if } x \geq y
\end{aligned}\right.
$$

where $e$ is the base of Naperian Logarithm. It is clear that $\circ$ is an AntiOperation on $X$ and $\therefore(X, \circ)$ is an AntiAlgebra.

(4) Let $X=\mathbb{R}$.

(i) $(X,+)$ and $(X, \times)$ are neither NeutroAlgebras nor PartialAlgebras.

(ii) For all $x, y \in X$ such that $x, y=0, \pm 1$, we have $x-y=y-x=0 \in X$ (True), otherwise for other elements, the result is False so that - is NeuroCommutative over $X$.

(iii) For all $0 \neq x, y \in X, x \div y \in X$ (True) (Inner) but for all $x \in X, x \div 0=$ indeterminate (Indeterminacy). It can be shown that $\div$ is NeutroAssociative over $X$. Hence, $(X, \div)$ is a NeutroSemigroup and therefore, it is a NeutroAlgebra which we call a NeutroField.

Let $\circ$ be a binary operation defined for all $x, y \in X$ by

$$
x \circ y=\left\{\begin{array}{rll}
-\sqrt{-1} & \text { if } x \leq y \\
\sqrt{-1} & \text { if } x \geq y
\end{array}\right.
$$

It is clear that $\circ$ is an AntiOperation on $X$ and $\therefore(X, \circ)$ is an AntiAlgebra.

(5) Let $X=\mathbb{C}$.

(i) $(X,+)$ and $(X, \times)$ are neither NeutroAlgebras nor AntilAlgebras.

(ii) For all $z, w \in X$ such that $z, w=0, \pm i$, we have $z-w=w-z=0 \in X$ (True), otherwise for other elements, the result is False so that - is NeutroCommutative over $X$.

(iii) For all $0 \neq z, w \in X, z \div w \in X$ (True) (Inner) but for all $z \in X, z \div 0=$ indeterminate (Indeterminacy). Therefore, $(X, \div)$ is a NeutroAlgebra which we call a NeutroField.

Let $\circ$ be a binary operation defined for all $z, w \in X$ by

$$
z \circ w= \begin{cases}i & \text { if } \quad|z|=|w| \\ j & \text { if } \quad|z| \leq|w| \\ k & \text { if } \quad|z| \geq|w|\end{cases}
$$

where $i j k=-1$. It is clear that $\circ$ is an AntiOperation on $X$ and $\therefore(X, \circ)$ is an AntiAlgebra.

Theorem 2.1. For all prime number $n \geq 2,\left(\mathbb{Z}_{n},+, \times\right)$ is a NeutroAlgebra called a NeutroField.

Proof. Suppose that $n \geq 2$ is a prime number. Clearly, 1 is the multiplicative identity element in $\mathbb{Z}_{n}$. For all $0 \neq x \in \mathbb{Z}_{n}$, there exist a unique $y \in \mathbb{Z}_{n}$ such that $x \times y=1$ (True) (T). However, for $0=x \in \mathbb{Z}_{n}$, there does not exist any unique $y \in \mathbb{Z}_{n}$ such that $x \times y=1$ (False) (F). This shows that $\left(\mathbb{Z}_{n}, \times\right)$ is a NeutroGroup. Since $\left(\mathbb{Z}_{n},+\right)$ is an abelian group, it follows that $\left(\mathbb{Z}_{n},+, \times\right)$ is a NeutroDivisionRing called a NeutroField. 


\section{Conclusion}

We have in this paper examined NeutroAlgebras and AntiAlgebras viz-a-viz the classical number systems $\mathbb{N}, \mathbb{Z}, \mathbb{Q}, \mathbb{R}, \mathbb{C}$ of natural, integer, rational, real and complex numbers respectively. In our future papers, we hope to study more algebraic properties of NeutroAlgebras and NeutroSubalgebras and NeutroMorphisms between them.

\section{Appreciation}

The authors are very grateful to all the anonymous reviewers for the useful comments and suggestions which we have found very useful in this work.

\section{References}

[1] Smarandache, F., Introduction to NeutroAlgebraic Structures and AntiAlgebraic Structures, in Advances of Standard and Nonstandard Neutrosophic Theories, Pons Publishing House Brussels, Belgium, Ch. 6, pp. 240-265, 2019.

[2] Smarandache, F., Introduction to NeutroAlgebraic Structures and AntiAlgebraic Structures (revisited), Neutrosophic Sets and Systems, vol. 31, pp. 1-16, 2020. DOI: 10.5281/zenodo.3638232.

[3] Smarandache, F., NeutroAlgebra is a Generalization of Partial Algebra, International Journal of Neutrosophic Science, vol. 2 (1), pp. 08-17, 2020. 